\title{
Sobre el
}

\section{modelo MOOC: filosofía, costo y estructura}

\section{Extracto:}

En un complejo panorama de puestos de trabajo que evolucionan constantemente y con la perspectiva, según el McKinsey Global Institute², de que para el año 2020 habrá unos 85.000 .000 de trabajos especializados (Shen, 2014), motivado todo ello por el rápido cambio que genera la tecnología, la formación está en un punto de inflexión importante. Con las universidades preparando a sus estudiantes en conocimiento enciclopédico, en competencias profesionales y en valores humanos, muchos son los que se preguntan cómo va a cambiar el método de aprendizaje en los próximos años, para así adaptarnos a los retos que nos plantean las nuevas tecnologías. Haciendo esa reflexión, algunos vieron a los MOOC como la panacea para lograr la disrupción en los entornos de enseñanza-aprendizaje, pero es verdad que se ha visto que esa disrupción no es tal desde el punto de vista metodológico. Aún se ven cursos MOOC que no generan el cambio tan esperado en el modo de aprendizaje basado en el receptor de la enseñanza, y hasta el propio director de una de las principales plataformas europeas de MOOC, llamada FutureLearn, ha realizado declaraciones comentando que los $\mathrm{MOOC}$ realmente no van a transformar la educación. Sea como sea, los llamados «nativos digitales» demandan nuevas formas de aprendizaje y hoy en día son los MOOC la avanzadilla con la que se está experimentando en nuevas formas de aprender con una educación basada en las nuevas tecnologías.

Palabras claves: aprendizaje virtual, MOOC, TIC, educación on-line, modelo de

Fecha de entrada: 30-03-2015

Fecha de aceptación: 10-04-2015

\footnotetext{
G. J. Palazio Arko, catedrático de Tecnología Audiovisual de la Universidad del País Vasco.

2 Dobbs, R. [2012]: The world at work: jobs, pay, and skills for 3.5 billion people. Disponible en: http://www.mckinsey.com/insights/employment_and_ growth/the_world_at_work.
} 


\section{About the} MOOC model: philosophy, cost and structure

\section{Abstract:}

In an increasingly complex of jobs that are constantly evolving, and with prospects, according to the McKinsey Global Institute, that by 2020 there will be about 85 millions killed jobs (Shen, 2014), all motivated by the rapid change generates technology, training is an important turning point. With universities preparing students for encyclopedic knowledge, skills and human values, many are wondering how it will change the method of learning the coming years, in order to adapt ourselves to new challenging technologies.

Some people saw the MOOC as a panacea for achieving in disruption of learning environments, but it is true that we have seen that this disruption is not such from a methodological point of view. There are $\mathrm{MOOC}$ that do not generate the change as expected in the learning mode based on the receiver of teaching. The director of a major MOOC european platforms call FutureLearn has made statements about the MOOCs are not going to transform Education.

In any case, the so-called «digital natives» demand new ways of learning and today the MOOC are the advance with which it is experienced in new ways learning with an education based on new technologies.

Keywords: e-learning, MOOC, ICT, online education, learning model, new technologies.

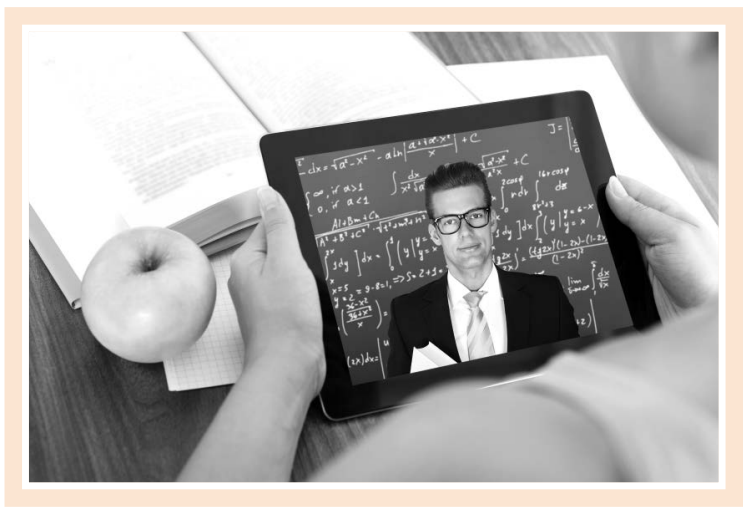

\section{SOBRE LA FILOSOFÍA MOOC Y SU LEGADO ACTUAL}

Los MOOC maduraron mucho durante 2014 y son bastantes las universidades que los han incorporado a sus programas de formación. En EE. UU., por ejemplo, 22 de las 25 universidades mejor situadas en los rankings ofrecen cursos gratuitos de este tipo (Shah, 2015). No obstante, la frontera entre algunos conceptos, aunque debe estar clara, no es comprendida por muchos educadores y formadores, al pensar que un MOOC de Udacity, por ejemplo, es realmente un MOOC. Muchas veces, usamos la palabra «MOOC» para denominar a los sistemas 0 a la tecnología que hace posible estos cursos, mientras que la metodología y esencia de este tipo de cursos se deja a un lado. $Y$ decimos esto porque para obtener en sentido estricto un $\mathrm{MOOC}$ se necesitan cinco componentes:

- Accesibilidad total al curso sin ningún tipo de barreras mientras dure el mismo (desde cualquier lugar y para cualquier persona).

- No debe haber cobro por la matrícula en el curso.

- Se debe dar libertad para que otros puedan reutilizar los materiales del curso.

- Hay que hacer uso de los formatos abiertos.

- Debe ir en plataformas de fácil uso para cualquier persona (usabilidad).

Si aplicamos estas normas, es evidente que los cursos de Udacity, por ejemplo, no cumplen con varias de las características MOOC: licencias libres de los materiales para que estos sean reutilizables y pago por los cursos. Así, Udacity cobra un mínimo de 200 dólares mensuales por un nanogrado (programas de entre 6 y 12 meses con 10 horas de dedicación semanal), título no oficial que está 
dirigido a preparar personas en los trabajos del siglo $X X{ }^{3}$. Esos cursos que se denominaban $\mathrm{MOOC}$ al principio y tenían su esencia en la filosofía MOOC han ido cambiando hasta convertirse en una especie de competencia profesional de la universidad tradicional.

Algunos expertos critican la forma de uso de los MOOC, y aunque la polémica está servida porque la revolución que iban a traer no ha sido tal, no cabe duda de que este tipo de cursos está enseñándonos muchas cosas. Una de ellas es el acercamiento a un modelo masivo y abierto de aprendizaje con nuevas formas de evaluación. Pero, tal vez, el mayor legado de los cursos MOOC ha consistido en que las universidades reciben una creciente presión para invertir más dinero en la enseñanza. Algunas universidades en EE. UU. llegan a gastar entre 39.000 y 325.000 dólares para cada MOOC que hacen. Este costo incluye las horas del personal dedicado y la alta calidad de la producción de los vídeos. Estos datos han sido recientemente publicados en la revista eCampus News (Stansbury, 2015). Muchas son las universidades que se están dotando de nuevas infraestructuras para ayudar a producir los cursos, contratando diseñadores expertos o inaugurando estudios de grabación para e-learning. Entre los datos del mencionado estudio, llevado a cabo por Fiona Hollands (Columbia University) y Devayani Tirthali (Brown University) sobre el costo de los $\mathrm{MOOC}^{4}$, destacan algunos de interés para calcular el coste de estos cursos (Stansbury, 2015):

- Todos los entrevistados que participaron en el desarrollo de un MOOC informaron del esfuerzo que conlleva dar a luz un MOOC, siendo ese esfuerzo «dos o tres veces mayor que la creación de un curso tradicional».

- Para crear 10 minutos de grabación de voz en vídeo de una lección a través de un programa de presentaciones como Impress o PowerPoint se requirieron entre 6 y 8 horas.

- Los cursos MOOC se consideraron más costosos en comparación con los tradicionales cursos on-line, debido a componentes $\mathrm{MOOC}$ específicos tales como el vídeo de alta calidad, las licencias de herramientas que sustituyen a las pruebas de los instructores y las tecnologías de aprendizaje entre pares.

\footnotetext{
${ }^{3}$ https://www.udacity.com/faq

${ }_{4}^{4}$ http://www.irrodl.org/index.php/irrod//article/view/1901/3069
}

\section{Algunos expertos critican la forma de uso de los MOOC, y aunque la polémica está servida, no cabe duda de que este tipo de cursos está enseñándonos muchas cosas. Una de ellas es el acercamiento a un modelo masivo y abierto de aprendizaje con nuevas formas de evaluación}

- Se percibió en los entrevistados consideraciones como la siguiente: «Se estiman cargas de trabajo del orden de 400 horas por cada miembro de la facultad que desarrollan el $\mathrm{MOOC}$, que es el equivalente al $26 \%$ de un año académico».

- Es posible que se tengan que aumentar y/o mejorar los servicios informáticos y de acceso a internet para los estudiantes que participan en cursos MOOC, por ejemplo, con el apoyo de personal de ayuda y modernizando los edificios y las redes para proporcionar suficiente capacidad de banda ancha a los estudiantes, los cuales necesitan un gran flujo de datos de forma simultánea en la descarga de ficheros audiovisuales.

- Sitios web institucionales y plataformas LMS deben proporcionar un punto de acceso a cursos MOOC de prestigio.

- Se tiende a involucrar a una serie de departamentos administrativos en actividades tales como la obtención de permisos de derechos de autor y el establecimiento de contratos entre la institución y el proveedor de la plataforma en línea, al igual que entre la institución y sus miembros docentes para hacer frente a los derechos de propiedad intelectual, el reparto de ingresos, la compensación a los profesores y la carga de trabajo que supone. Otras regulaciones como las de cumplimiento y no cumplimiento de contrato también deben ser consideradas.

- Para las instituciones que conceden créditos en los MOOC, las admisiones de los estudiantes, su registro, la autentificación de los pagos en estos cursos y los sistemas de acreditación son procesos que deben estar incluidos en los sistemas de inscripción en la plataforma. 
Hablando de costos, recordemos que los cursos multimedia de los años noventa del siglo pasado también eran percibidos al principio como una forma de ahorrar dinero, algo que muchos creyeron a rajatabla y que posteriormente se puso en cuestión. Hacer un curso multimedia enlatado para una duración media de dos años generaba muchas expectativas en lo correspondiente al ahorro de costes en la enseñanza, pero, finalmente, como mencionó Philip Barker (Bartolomé, 2013), se demostró que la generación de aquellos cursos multimedia era más costosa que la propia enseñanza tradicional cara a cara. Retrocediendo incluso a la década de los sesenta, como menciona Jeffrey Young (2013), se predecía un sistema parecido a lo que hoy vemos con los MOOC o las conferencias TED y que consistiría en una especie de vídeo-enciclopedia actualizada para que cualquier estudiante pudiera estar al día de cualquier conocimiento. Todo eran ideas de lo que en un futuro se debía generar en modo sostenible, porque no cabe duda de que el éxito solo lo da la sostenibilidad de los sistemas y proyectos. Ni siquiera la Wikipedia se libra de ello, ya que la financiación de esa inmensa biblioteca on-line es aún hoy en día generadora de muchos quebraderos de cabeza.

Un proveedor de cursos MOOC como Coursera ha experimentado con varias formas de generar dinero a través de los MOOC, y realmente parece que ha encontrado una rentable. Esta forma de generación de beneficios monetarios que ya están sobre el millón de dólares mensuales se fundamenta en la certificación autentificada, que tiene por estudiante un costo de entre 30 y 70 dólares según el tipo de curso (Shah, 2014). Esa búsqueda de nuevos modelos de certificación es otra de las características de los MOOC, que siguen investigando en esa vía por medio de medallas o badges informales, certificación con empresas partners 0 acreditación vocacional otorgada por los proveedores de contenido (De Waard, 2014).

El fenómeno de los MOOC sigue generando todo tipo de críticas y análisis en el campo de la educación. De hecho, se está haciendo una apuesta importante en Europa, sobre todo por atisbar el futuro de este tipo de cursos que en los últimos tres años han generado tantas expectativas. Teniendo en cuenta que son uno de los recursos de educación abierta del campo de la transmisión de conocimiento, se presentan como algo novedoso y que algunos hemos comparado con lo que ha supuesto la Wikipedia al campo del conocimiento enciclopédico. Si en el espacio que hoy llena la Wikipedia ya no hay hueco para las enciclopedias comerciales, muchos estábamos a la espera de que apareciera algo tan rupturista con el pasado como la Wikipedia, pero en este caso algo que se ciñese al campo de la transmisión de conocimiento, en vez del espacio del conocimiento pasivo-acumulado y enciclopédico. Es decir, una herramienta y metodología que sirviera para dar a conocer cómo los ciudadanos iban a aprender en el futuro. Desde Canadá, George Siemnes y Stephen Downes nos dieron la opción de poder empezar a soñar con algo diferente a la clase de siempre y además con unas metodologías que incidían en el aprendizaje colaborativo y rico gracias a los recursos que hoy en día se generan en internet por la revolución que supuso la web 2.0 de lectura y escritura. A principios de marzo de 2015 el panorama europeo de los $\mathrm{MOOC}$ en cuanto a número de cursos que existen en diferentes países es de 1.066 según el portal de la Comisión Europea de Educación Abierta, con una distribución que podemos ver en la siguiente figura:

\section{Figura 1. Distribución de cursos MOOC por países en parte de Europa}

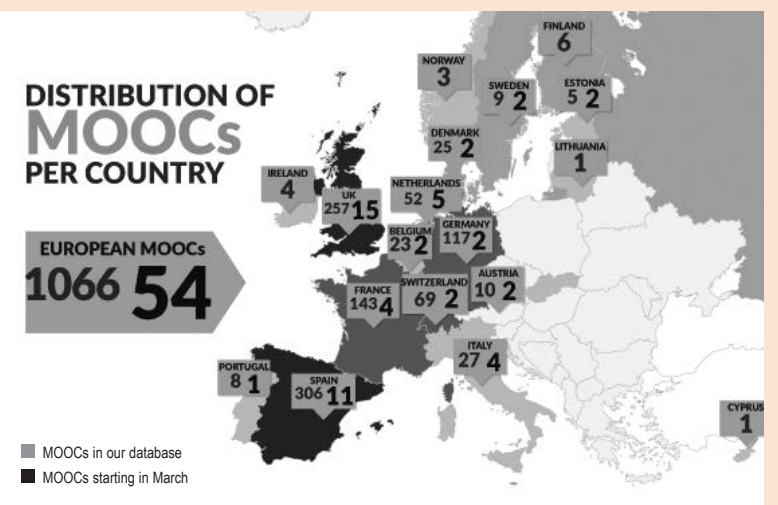

Fuente: http://openeducationeuropa.eu/en/news/new-download-european-moocscoreboard-data-one-click. 
Por otro lado, el tema de los nanogrados de Udacity está siendo algo más que un experimento interesante, ya que es una nueva forma de atraer a los trabajadores de la industria. Udacity, que nace como plataforma para cursos MOOC y que con el paso del tiempo se quiere convertir en la universidad del polo tecnológico de Sillicon Valley, lanzó en octubre de 2014 sus primeros nanogrados que ahora están llevando la certificación digital de la empresa Accredible ${ }^{5}$. Es la búsqueda de certificación válida para unos trabajadores de la industria tecnológica que van a necesitar una continua formación de por vida, algo en lo que las plataformas de cursos MOOC pueden ayudar convirtiéndose en la plataforma básica a usar. Udacity ha empezado a lograr que importantes organizaciones de Sillicon Valley den su validación a los nanogrados, y ello marca un camino que aún está por implantar en otros polos tecnológicos del mundo.

En febrero de 2015 se ha publicado el informe sobre las ideas que tienen las instituciones universitarias europeas respecto a los MOOC. En la encuesta, dirigida por los profesores Darco Jansen y Robert Schuwer durante los tres últimos meses del año 2014, se destaca que las universidades europeas tienen una consideración diferente del fenómeno de estos cursos masivos abiertos respecto a la opinión que tienen en los EE. UU.

La encuesta llevada a cabo en centros universitarios que abarcan un total de unos 2,8 millones de estudiantes de 22 países europeos se basó en preguntas idénticas usadas en encuestas ya realizadas en $\mathrm{EE}$. UU. durante los años 2013 y 2014. Los resultados de esta encuesta han mostrado grandes diferencias entre Europa y Norteamérica, siempre referenciando a Europa en su nivel geográfico-cultural y no político, ya que no solo naciones de la Unión Europea respondieron a la encuesta.

Una de los resultados más llamativos es el de la respuesta de las instituciones educativas europeas en el sentido de que no ven situación problemática en el futuro entre la titulación otorgada por los MOOC y la titulación universitaria como la conocemos ahora. En EE. UU. es un porcentaje mayoritario el que cree que se va a dar esa problemática en el futuro. Podemos llegar a pensar que en Europa las instituciones son más conservadoras y les cuesta creer más en los cambios, en comparación con lo que sucede en EE. UU. Las instituciones universitarias estadounidenses ven a los MOOC también

\footnotetext{
5 http://www.accredible.com
}
Las instituciones universitarias estadounidenses ven a los MOOC como una herramienta muy eficaz para fichar y promocionar a los mejores estudiantes y profesores, mientras que en Europa no se está apostando por el reconocimiento del gran trabajo que lleva la preparación de cursos impartidos como MOOC

como una herramienta muy eficaz para fichar y promocionar a los mejores estudiantes y profesores, mientras que en Europa no se está apostando por el reconocimiento del gran trabajo que lleva la preparación de cursos impartidos como MOOC. Son formas diferentes de mirar el campo de los MOOC, que sin duda están siendo la avanzadilla del cambio hacia una transmisión de conocimientos en forma abierta y masiva.

\section{SOBRE LA ESTRUCTURA Y LA INTERFAZ DE LOS MOOC}

Los MOOC, al ser cursos, tienen de alguna forma una estructura, con un temario predeterminado y unos objetivos de aprendizaje en la mayoría de los casos, con las excepciones de los MOOC que con aprendizaje rizomático puedan establecer carencia de estructuras que no dificultan el aprendizaje, pues el componente principal en esos MOOC rizomáticos es el aprendizaje colaborativo acompañado de expertos que van generando caminos de aprendizaje insospechados al inicio del curso. Esta estructura de los $\mathrm{MOOC}$ no rizomáticos, no obstante, no tiene por qué ser contradictoria con la enseñanza modular que busca la rentabilización de los materiales generados y de gran costo. Usar un módulo de varias horas en una asignatura puede ser válido para otra materia que necesite de esos contenidos en su senda curricular. Se produce ya una tendencia a la modularización de los materiales de aprendizaje, que al fin y al cabo, en muchos casos, ya se 
han convertido en recursos abiertos de aprendizaje (OER, en inglés). Los MOOC deben ser capaces de generar continuamente nuevos materiales de aprendizaje teniendo en cuenta la renovación constante que se produce cada vez a mayor velocidad en el campo del conocimiento aplicado. Incluso los foros de instructores y estudiantes deben ser una fuente de regeneración y servir de faro 0 de guía para la búsqueda de recursos más actuales y motivadores. El éxito de los MOOC estará en gran medida en ello; es decir, en crear industrias generadoras de contenido continuamente.

Por lo tanto, la idea de que se debe conseguir que los materiales de los MOOC no caigan en desuso 0 queden obsoletos en poco tiempo es una de las cuestiones que están sobre la base de este tipo de aprendizaje. Creo que la metodología de la renovación de materiales por curso o mientras dure el curso MOOC es uno de los temas más importantes dentro de la implantación de este tipo de cursos. Hasta ahora, una de las pocas formas que tenemos de asegurar que los materiales sirvan para su cometido es la de poner fechas de finalización de trabajos, algo que se hace en la mayoría de los MOOC; incluso en los MOOC que tienen más de aprendizaje rizomático en vez de aprendizaje estructurado. Parece que la idea de la motivación gracias a las fechas de finalización seguirá estando todavía en la polémica que envuelve a los MOOC, con los partidarios de esa idea y los contrarios, que opinan que los recursos de aprendizaje sin fecha de finalización no tienen por qué quedar en desuso y no ser parte del futuro aprendizaje (Parkingson, 2014).

\section{Los MOOC deben ser capaces de generar continuamente nuevos materiales de aprendizaje teniendo en cuenta la renovación constante que se produce cada vez a mayor velocidad en el campo del conocimiento aplicado}

En esa estructura, el formato y la interfaz van de la mano. A la hora de apuntalar con éxito un curso MOOC, la estructura debe ser comprensible y simple para el estudiante, siempre haciendo hincapié en los objetivos del aprendizaje. Esas metas que debe tener el alumno parece que pueden ser bien implementadas simplemente con vídeos de alta calidad (screencasts, vídeo con profesor, vídeo sin profesor, vídeos híbridos) y ejercicios de preguntas-respuesta; pero lo que siempre debe aparecer son los objetivos del aprendizaje en cada uno de los módulos o unidades del MOOC. En la figura 2 se puede ver un buen ejemplo de interfaz de usuario para poder comprender la simplicidad y efectividad que se logra con la implantación de este tipo de interfaz MOOC.

Por último, tenemos que mencionar la importancia de las evaluaciones y ejercicios de práctica de última generación, que cobran realidad en el campo de los MOOC con los ORA (open response assessment). Ya se ha repetido muchas veces que los ejercicios y los vídeos son los componentes claves en la estructura de la mayoría de los MOOC. Estos tipos de ejercicios tienen sus ventajas y desventajas en lo correspondiente a su implantación en el sistema de Open Edx, siendo este un campo en el que aún hay mucho por recorrer. Las tres grandes ventajas de este tipo de ejercicios de corrección por máquina y algoritmos son:

- Son beneficiosos para los estudiantes en sus procesos de autoaprendizaje.

- Son herramientas que dan más rapidez que la corrección por pares.

- Puedan dar un feedback ilimitado de datos sobre gramática y pronunciación.

Entre las desventajas de los ORA hay dos claras de ver:

- Esta tecnología no ha sido lo suficientemente testada en datos en vivo.

- No puede dar una retroalimentación de una cualidad humana.

Los módulos de evaluación ORA de Edx están albergados en GitHub ${ }^{6}$ y abarcan, bajo la sigla ORA, en su segunda versión, ORA2, el aprendizaje por máquina, con instructor, por pares, autoevaluación y chequeo básico.

\footnotetext{
${ }^{6}$ https://github.com/edx/edx-ora2
} 


\section{SOBRE LOS MODELOS DE SOSTENI- BILIDAD DE LOS MOOC}

El especialista británico en tecnología educativa Steve Parkinson diferencia de modo inteligente entre los aprendices cash poor/time poor y los cash rich/time poor, es decir, entre los aprendices adinerados que pueden pagar por un curso y tienen poco tiempo para realizarlo y los que no tienen demasiado dinero y tienen también poco tiempo para llevarlo a cabo. Al final es posible que el modelo disruptivo venga por el lado de las plataformas de micropago de aprendizaje a lo largo de la vida que faciliten a los dos grupos de ciudadanos el aprendizaje continuo sin las cortapisas de las tareas a realizar en un tiempo determinado. Detrás de ese modelo flexible para el ciudadano que quiere aprender hay varias plataformas con muchos usuarios. Eso es algo que están demostrando plataformas como Treehouse, Lynda, Codecademy o Udacity.

En el ambicioso proyecto Treehouse, realizado con financiación de Google, entre otros, la metodología de aprendizaje con tecnología sigue los pasos de los MOOC, aunque rompe con el modelo abierto desde el momento en que es un servicio de subscripción con micropago. Con la cuota mensual de 25 dólares, Treehouse da acceso a una serie de cursos de programación con una visión de acceso y desarrollo dirigido por uno mismo, para que cada cual pueda ir controlando su aprendizaje sin las cortapisas del tiempo, en diversas tecnologías y áreas del diseño y la programación. Al contrario de los MOOC con tiempos específicos para las tareas, en esta plataforma el usuario no tiene el agobio que puede suponer la realización de una tarea en un periodo corto de tiempo. La simplicidad del interfaz de Treehouse da la opción a que el modo de autoaprendizaje pueda tener éxito y las tareas se vayan haciendo poco a poco. Es un modelo de aprendizaje por subscripción en el que los usuarios son los que marcan los tiempos de su propio aprendizaje y consiguen reconocimiento por ello una vez realizadas las tareas. Así pues, tenemos tres modelos diferentes que se sirven de la tecnología de los MOOC y que buscan rentabilizar la preparación de materiales, algo que tiene un coste importante cuando se hace bien:

- Modelo abierto de pago por reconocimiento de realización del curso (Coursera).

- Modelo cerrado de pago por curso único (Udemy).

- Modelo cerrado de pago por subscripción a todos los cursos de la plataforma (Treehouse).

De los tres modelos, el pago por subscripción a todos los cursos, al igual que pasa con el consumo de la música o del cine, es una de las opciones que se ve con más futuro. Incluso la plataforma francesa OpenClassrooms se ha apuntado al modelo de subscripción premium mensual. Esta plataforma líder en el Estado francés ha sido recientemente elegida por el presidente François Hollande para dar formación y certificación a todas

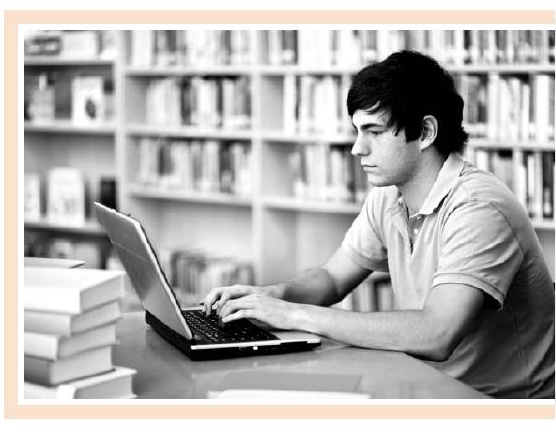

las personas que buscan trabajo en Francia a partir de septiembre del año 2015․ Como se ve, se trata de abrir una gran puerta a personas sin empleo para que tengan acceso a cualquier tipo de certificación de OpenClassrooms con un modelo de acceso premium gratuito. No cabe duda de la importancia de la apuesta que hace el Gobierno francés en el campo de la formación profesional.

Al fin y al cabo, si una plataforma on-line de cursos consigue una especialización en un campo del conocimiento, se puede empezar a probar con sacar provecho al modelo, ya que todos los cursos afines se muestran como una gran opción al usuario que busca una especialización en su trabajo. El tener acceso a cualquier curso de una plataforma es una forma de asegurar el modelo financiero de la propia plataforma una vez se tengan suficientes usuarios y cursos atractivos sobre las materias que interesan.

Con plataformas tipo MOOC también han aparecido los denominados SPOC (small private on-line courses) $)^{8}$, una versión no abierta y

\footnotetext{
7 http://goo.gl/9oesWh

8 http://en.wikipedia.org/wiki/Small_private_online_course
} 
Figura 2. Interfaz de un curso en Treehouse

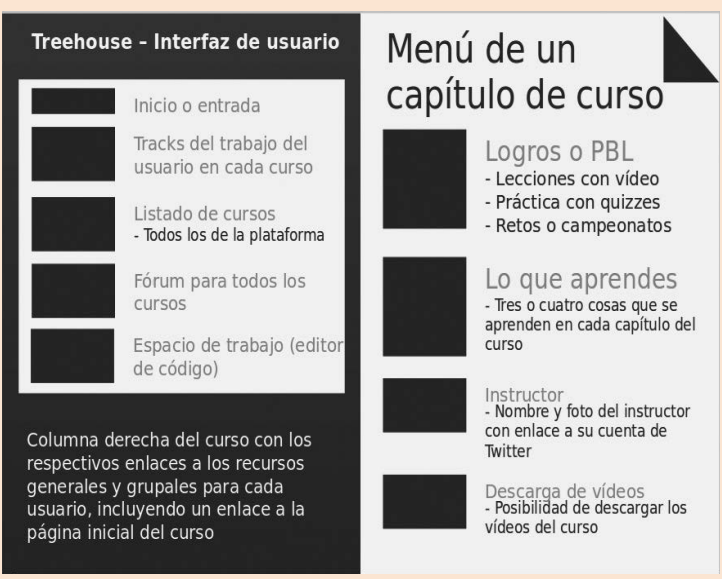

Fuente: figura realizada por G. J. Palazio Arko.

privada que se está experimentando con los estudiantes presenciales de campus universitarios, aunque también se usa ya en entornos de formación especializada con clases reducidas de 17-20 personas (Goral, 2013). Los SPOC nacen en el año 2013 de la mano del profesor Armando Fox en la Universidad de Berkeley en California. Se trata de un modelo de sostenibilidad del tipo negocio-a-negocio en el que las empresas pueden encontrar una forma de hacer sostenible la costosa creación de materiales ricos expuestos en potentes plataformas MOOC. En el campo universitario, un SPOC permite dar créditos a los estudiantes dentro de un curso. Con contenido interactivo y evaluación puesta por el profesor, sirven al alumno para que, controlando su propio tiempo, pueda superar una parte de la materia de un curso. Los SPOC dan soporte al blended learning 0 aprendizaje mixto y a la clase invertida o flipped classroom, dos formas de aprender muy de actualidad hoy en día. Sea como fuere, el modelo SPOC tiene una gran ventaja para su implantación en los entornos de formación por la gran usabilidad y potencia de su plataforma, heredada de los MOOC. Ello los hará muy apetecibles para los entornos empresariales en donde el pago por la creación de buenos materiales está asegurado.

Otras formas que se han experimentado desde el concepto de cursos MOOC es la de los DOCC (distributed open collaborative course) y los MOOR o investigaciones masivas abiertas on-line (Pernías y Luján, 2013). Pero aún ninguna de ellas ha cogido la suficiente popularidad para hacer de ella un modelo sostenible y generalizado para los entornos de enseñanza-aprendizaje e investigación.

\section{BIBLIOGRAFÍA}

Bartolomé, A. [2013]: «Qué se puede esperar de los MOOC», Comunicación y Pedagogía, núm. 269-270, págs. 49-55.

De Waard, I. I. [2014]: MOOC yourself. Set up your own MOOC for business, non-profits, and informal communities. Autoedición de la autora, 2. ${ }^{\mathrm{a}}$ versión del original publicado en 2013.

Goral, T. [2013]: «SPOCs may provide what MOOCs can't», UB University Business. Disponible en: http:// www.universitybusiness.com/article/ spocs-may-provide-what-moocscan $\%$ E2\% $\% 0 \% 99$ t.

Parkingson, S. [2014]: Do dead lines improve MOOCs? Disponible en: https://steveparkinson101.wordpress.com/2014/03/17/do-deadlines-improve-moocs.

Pernías, P. y Luján, S. [2013]: «Los MOOC: orígenes, historia y tipos», Comunicación y Pedagogía, núm. 269-270, págs. 41-48.

Shah, D. [2015]: MOOC Watch Jan 2015: on-line courses raise their game. Disponible en https://www.class-central. com/report/moocwatch-jan-2015-online-courses-raise-game.

[2014]: How does coursera make money? Disponible en: https://www.edsurge.com/n/2014-10-15-how-doescoursera-make-money.

Shen, C. [2014]: Announcing nano degrees: a new type of credential for a modern work force. Disponible en: http:/l blog.udacity.com/2014/06/announcingnanodegrees-new-type-of.html.

Young, J. R. [2013]: Beyond the MOOC hype: a guide to higher education's high-tech disruption, Washington DC, EE. UU., The Chronicle of Higher Education. 